Annuaire suisse de politique de développement

3 | 1983

Annuaire Suisse - Tiers Monde 1983

\title{
II. Politique économique extérieure
}

Hilar Stetter, Jacques Martin et Philippe Besson

\section{OpenEdition}

\section{Journals}

Édition électronique

URL : http://journals.openedition.org/aspd/1034

DOI : $10.4000 /$ aspd.1034

ISSN : 1663-9669

\section{Éditeur}

Institut de hautes études internationales et du développement

\section{Édition imprimée}

Date de publication : 1 janvier 1983

Pagination : 87-99

ISSN : 1660-5934

\section{Référence électronique}

Hilar Stetter, Jacques Martin et Philippe Besson, « II. Politique économique extérieure », Annuaire suisse de politique de développement [En ligne], 3 | 1983, mis en ligne le 06 décembre 2012, consulté le 08 septembre 2020. URL : http://journals.openedition.org/aspd/1034 ; DOI : https://doi.org/10.4000/ aspd. 1034 


\section{POLITIQUE ECONOMIQUE EXTERIEURE}

\section{GARANTIE CONTRE LES RISQUES A L'EXPORTATION ET GARANTIE CONTRE LES RISQUES A L'INVESTISSEMENT}

La garantie fédérale contre les risques à l'exportation a bouclé son exercice 1982 avec un excédent de dépenses, une fois de plus. II y en a pour 186,54 millions de francs. Le déficit a été comblé grâce à la liquidation des réserves, soit 77 millions de francs. La GRE fournit aux exportateurs suisses une garantie contre les risques politiques, monétaires et de fabrication.

Le total des compensations pour pertes subies s'est élevé à 360 millions de francs en 1982. 178,8 millions de francs concernent des pertes de change, 97,1 millions de francs des consolidations de dettes accordées à la Turquie, au Togo, au Sénégal et à Madagascar et 84 millions de francs des risques d'ordre politique $(17,7$ millions de francs en 1981).

En 1982, les recettes $n$ 'ont été que de 175,3 millions de francs, dont 121 millions en taxes perçues. Le total des engagements contractés était à fin 1982 de 19,7 milliards de francs, légèrement en recul par rapport à l'année précédente du fait de la baisse du montant des garanties contre les risques monétaires. La majeure partie des engagements contractés concerne l'industrie des machines. L'an passé, de nouvelles garanties ont été demandées pour un montant de 7,5 milliards de francs.

L'augmentation des dépenses s'explique par une forte progression des risques de nature politique, eux-mêmes engendrés par le déséquilibre de la balance des paiements des pays importateurs. En cas de déséquilibre persistant de la balance des paiements, on tente de consolider les dettes. Les risques monétaires représentent près de la moitié du déficit de la GRE. On ne sait pas encore comment assainir ce volet de la GRE et l'industrie d'exportation, dont les affaires fléchissent, refuse de nouvelles restrictions. A la fin du mois de mars, le Conseil fédéral a d'abord prolongé la possibilité de s'assurer contre les risques monétaires. En juin, il a augmenté les primes de l'assurance contre les pertes de change. Le niveau des prestations reste inchangé. Taxes et franchise ont été relevées au 1er juillet 1983, la franchise étant portée de $5 \%$ à un maximum de $12 \%$. 
Total des montants garantis

Garanties concernant I'ensemble des

pays en développement

Garanties concernant les pays les

plus pauvres

Excédent de dépenses

0,187

GRE et pays en développement

A fin $1982,69,1 \%(65,7 \%$ en 1981$)$ des garanties accordées concernaient des pays en développement. Elles s'élevaient à 13,6 milliards de francs $(14,6$ milliards en 1981). Leur répartition: $34,9 \%$ concernaient des pays asiatiques, $23,1 \%$ des pays latino-américains, $26,8 \%$ des pays africains et $15,3 \%$ des pays européens en développement. La GRE a assumé pour 3,1 milliards de francs de risques en Argentine, au Brésil et au Mexique.

Les 67 pays en développement à bas revenu (selon les critères de I'OCDE) envers lesquels la Suisse accorde sa garantie compte tenu des principes de la coopération au développement ont mobilisé $11,7 \%$ du montant total des garanties. En chiffres absolus, 2,3 milliards de francs. La moitié de ce montant total ne concerne que l'Egypte, le Honduras, l'Inde et l'Indonésie. De nouvelles garanties s'élevant en tout à 500 millions de francs ont été accordées l'an passé pour des exportations à destination de ces quatre pays. Les exportations vers l'Egypte et I'Indonésie ont bénéficié de garanties supérieures à 50 millions de francs.

Deux grandes commandes ayant fait l'objet d'une demande de garantie ont prêté à discussion. Losinger SA, entreprise de construction, a demandé qu'on couvre les risques de l'édification du barrage de Manantali, au Mali. Les travaux sont devisés à 165 millions de francs. La Banque mondiale a refusé de financer ce projet dont les plans ont été établis par un consortium international. La Banque et d'autres institutions multilatérales de développement ont fait observer que le projet aurait des conséquences socio-économiques négatives et que les Etats concernés étaient fortement endettés. Les œuvres d'entraide privées estiment que cette demande de garantie permettait de tester l'applicabilité des principes de notre politique de développement quand il s'agit de se prémunir contre les risques à l'exportation vers les pays les moins avancés. 
Une grosse commande de 1,7 milliard de francs passée aux firmes Sulzer-Escher-Wyss et BBC pour équiper I'usine hydro-électrique Atatürk en Turquie requiert également l'octroi de la GRE. Répondant à une demande du conseiller national Jaeger (Ind/SG), le conseiller fédéral Honegger a fait savoir en décembre 1982 que les seuls intérêts des capitaux mobilisés s'élèveraient à 700-800 millions de francs couverts par la GRE. Le conseiller fédéral Honegger estima que, dans ce cas, les risques étaient très sérieux et qu'il fallait se garder de créer un précédent.

Dans l'intervalle, la garantie allemande contre les risques à l'exportation Hermès prit à son compte une partie de la commande. Le Conseil fédéral suivit en juin 1983 en autorisant la GRE à couvrir un montant de $\mathbf{5 0 0}$ millions de francs pour cette commande. Deux motions présentées au Conseil national demandèrent en outre que les bénéfices de la Banque nationale fussent, en partie au moins, consacrés au financement de la GRE. La fraction Ind/PDC souhaite mettre une partie des bénéfices à la disposition de la GRE, alors que le conseiller national Feigenwinter (PDC/BL) veut que les bonis servent à financer des prêts à la GRE et les pertes enregistrées sur les risques de change. Le Conseil fédéral a accepté ces deux motions sous forme de postulats.

\section{La garantie contre les risques de l'investissement}

D'une enquête de I'OFAEE sur la nécessité de la GRI, il résulte que cet instrument ne joue qu'un rôle mineur dans la plupart des décisions d'investir. Les chiffres ne font que le confirmer. En 1982, la GRI n'a été requise que pour trois investissements représentant un total de 1,6 million de francs. Les garanties allouées atteignaient à fin 1982 un total de 43,6 millions de francs, dont 12 millions pour le relèvement d'une garantie déjà accordée en 1981. Aucune compensation pour perte subie n'a dû être versée. La fortune totale de la Suisse à l'étranger a été estimée en 1981 à 39 milliards de francs, les investissements directs dans les pays en développement à 6 milliards de francs 13,5 milliards en Amérique latine, 1,3 milliard en Europe méridionale, 0,5 milliard en Asie et 0,5 milliard en Afrique).

\section{Sources}

EVD - Communiqué de presse 21.6.1983.

Rapport 1982 du Conseil fédéral.

Bulletin sténographique de l'Assemblée fédérale 1982, Conseil national pp. 751 ss. et pp. 1670 ss.

NZZ, 16.8.1982, 24.3-3.6.1983.

TA, 21.6.1983.

i3m-actualités $3 / 1983$. 


\section{ACCORD SUR LA PROTECTION DES INVESTISSEMENTS}

Le Conseil fédéral a publié en novembre 1982 son message concernant l'accord sur la protection des investissements. II y demande au Parlement de prolonger à nouveau de dix ans la validité de l'arrêté pris à ce sujet en 1983. Le Conseil des Etats a ratifié cette demande lors de sa session de printemps et le Conseil national au cours de sa session de l'été 1983. S'il n'est pas fait usage du référendum, le texte entrera en vigueur en février 1984. L'arrêté autorise le Conseil fédéral à conclure d'autres accords semblables à I'avenir.

Depuis 1961, la Suisse a conclu 34 accords sur la protection des investissements, dont 23 avec les pays africains, 7 avec des pays d'Asie, 3 avec des pays de l'Amérique latine et 1 avec Malte.

Ces accords ont pour but de protéger les investissements suisses à l'étranger, notamment dans les pays en développement. La protection juridique des investissements s'en trouve fortifiée et le climat propre aux investissements en est amélioré. Le recours à des capitaux provenant de l'économie privée, aux investissements par exemple, est l'une des formes de la coopération au développement fournie par la Suisse. Au demeurant, l'octroi d'une garantie des risques encourus par un investissement peut dépendre de l'existence d'un accord avec le pays concerné.

La Suisse figure au quatrière rang des pays qui investissent à l'étranger. Selon une estimation des banques, le montant total des investissements suisses à l'étranger s'élevait en 1981 à 39 milliards de francs.

Lors de la discussion du message au Conseil des Etats, on présenta ainsi les critères à observer lors de la conclusion de nouveaux accords:

1) Une situation économique relativement stable;

2) I'existence de certaines dispositions et garanties juridiques, touchant notamment les expropriations ou mesures de nationalisation;

3) une réglementation du statut de la nation la plus favorisée qui soit applicable aux investisseurs nationaux et étrangers;

4) des dispositions sur les transferts de capitaux avec des précisions concernant les contrats, livraisons, règlements des droits de brevet ou produits de liquidations de capitaux;

5) des clauses concernant le règlement des litiges.

Au cours de la discussion au Conseil national, Jean Ziegler (PS/GE) n'admit pas qu'on puisse considérer comme aide au développement les investissements privés dans les pays en développement, puisqu'ils contribuent à leur endettement. Le Service d'information tiers monde s'éleva contre le fait qu'on considère généralement que les investissements privés servent le développement et réclama une aide sélective en faveur des investissements conformes aux principes de la politique suisse de développement. 


\section{Sources}

Bbl 1982 III, pp. 1025 ss.

Bulletin officiel de I'Assemblée nationale 1983, Conseil des Etats p. 158; Conseil national pp. 701 ss.

i3m-actualités 1/1983.

\section{ACCORDS DE DOUBLE IMPOSITION}

Un accord de double imposition a été conclu le 11 janvier 1983 à Colombo, entre la Suisse et le Sri Lanka, troisième pays en développement à signer un tel accord avec la Confédération, après Trinité-etTobago et la Malaysia. Ces accords ont pour but d'éviter la double imposition du revenu et de la fortune. Les Etats intéressés renoncent de part et d'autre à certaines rentrées fiscales.

Ces derniers temps, le Sri Lanka a déployé maints efforts pour conclure des accords de double imposition. Ce pays souhaite en effet devenir plus attrayant aux yeux des investisseurs étrangers. II se montrait prêt à diverses concessions en faveur de la Suisse, allant jusqu'à renoncer à l'application du droit national selon les cas.

La Suisse a intérêt à conclure de tels accords pour préserver sa capacité internationale de concurrence et pour faire bénéficier ses investissements d'une sécurité accrue. Elle prend exemple sur les modèles élaborés par l'OCDE et insiste pour qu'on prenne en considération les services, les gains des entreprises, les brevets, les intérêts et dividendes ainsi que les impôts à la source. L'accord avec le Sri Lanka est généralement conforme au droit fiscal en usage en Suisse et représente pour notre pays un modèle profitable. II est conforme aux lignes directrices de la politique de développement tracées dès 1973 pour des accords de ce genre.

Une interpellation Augsburger (UDC/BE) a fourni au Conseil fédéral I'occasion de préciser sa politique en la matière. La Suisse est prête à signer un accord de double imposition à condition que les relations économiques bilatérales se développent favorablement. Certains pays de I'OCDE (République fédérale allemande, Grande-Bretagne) ont de nombreux accords de double imposition avec des pays en développement. Ce n'est pas le cas de la Suisse. Anglais et Allemands connaissent de forts impôts à la source; c'est à partir de ce plafond qu'il leur est assez facile de faire des concessions aux pays en développement. Le niveau des impôts à la source est bas en Suisse, les mêmes concessions ne sont pas possibles. Les cantons n'y consentiraient pas. Les accords de double imposition n'en complètent pas moins l'éventail des mesures de politique commerciale de la Suisse en favorisant les échanges et les investissements. Des négociations avec d'autres pays en 
développement (Argentine, Brésil) et des pourparlers avec l'Arabie saoudite et le Nigeria n'ont pas encore amené la conclusion d'autres accords de double imposition.

\section{Source}

Bbl 1983, p. 1168.

Bulletin sténographique de l'Assemblée fédérale, Conseil national 1982, pp. 1809 ss (82.553 interpellation Augsburger).

NZZ, 26-27.3.1983.

\section{ACCORDS DE REECHELONNEMENT DES DETTES}

Selon les recommandations du Club de Paris, dont la Suisse fait partie, la Suisse a conclu des accords de rééchelonnement des dettes avec divers pays débiteurs. Trois accords en sont résultés, qui sont entrés en vigueur entre juillet 1982 et juin 1983 .

Le 4 novembre 1982 est entré en vigueur l'accord conclu en 1981 avec la République centrafricaine pour le report d'une dette de 19 millions de francs en intérêts et capital échéant au 31.12.1981 et restée impayée. La dernière tranche de la dette doit être versée au plus tard le 30 juin 1990.

Le 12 octobre 1982 est entré en vigueur l'accord sur le report de dettes malgaches résultant de crédits à l'importation d'un montant de 4 millions de francs et dont l'échéance a été nouvellement fixée au 30 septembre 1991.

Enfin, entrée en vigueur le 19 octobre 1981 d'un accord du même type ayant pour but de rééchelonner des dettes soudanaises résultant de crédits à l'exportation d'un montant de 21 millions de francs. Les dettes sont à régler au plus tard le 1er juillet 1992 .

\section{Sources}

SA 1982, pp. 2021 ss, p. 2091, p. 2095.

\section{PREFERENCES DOUANIERES}

$16 \%$ des livraisons faites par des pays en développement sont entrés en Suisse au bénéfice des préférences douanières en 1982. Comme il ressort du tableau ci-dessous, la part de ces importations au total des arrivages en provenance des pays en développement est donc restée stable depuis 1980. En fait, des marchandises plus nombreuses auraient dû bénéficier des préférences douanières, car les dispositions existantes n'ont été utilisées qu'à $38 \%$ en 1982, tendance à la hausse depuis 1980 . 


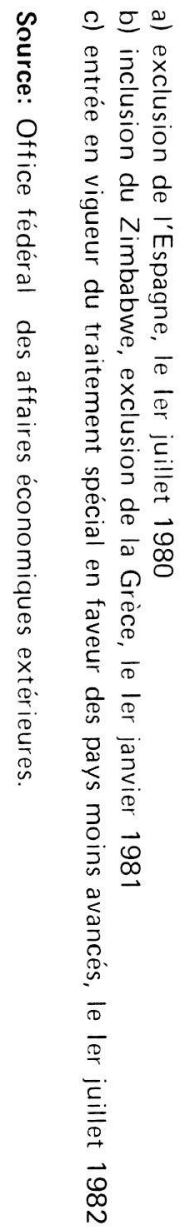

$\begin{array}{lll}\overrightarrow{0} & \overrightarrow{0} & \vec{\varphi} \\ \stackrel{\infty}{N} & \stackrel{0}{0} & 0 \\ \bar{\Xi} & \vec{\sigma} & \underline{\omega}\end{array}$

\begin{tabular}{lll}
$G$ & 0 & \multicolumn{1}{c}{} \\
0 & 0 & 0 \\
0 & 0 & 0 \\
8 & 0 & 0 \\
0 & 0
\end{tabular}

छ

Année

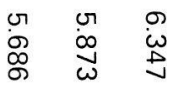

$\bar{\omega}$

Importations en prove-

Volume total des importations de la

$\bar{N}$ Suisse

en millions de francs

nance des pays en déve-

loppement (PVD) a)

en millions de francs

$\sigma \quad \omega v$

$\begin{array}{lll}\infty & 0 & 0 \\ \infty & \infty & \infty\end{array}$

Part des importations en provenance des PVD en $\%$ de la colonne (3) par rapport à la colonne (2)

$N$
$\omega$
$\sim$

G

Importations pouvant bénéficier du traitement préférentiel en millions de francs

$\begin{array}{lll}\infty & \infty & \wp \\ \mathscr{C} & \infty & \infty \\ \sigma & & \wp\end{array}$

$\bar{\sigma}$

Importations ayant effectivement bénéficié des préférences

en millions de francs

Degré d'utilisation des

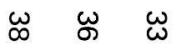
avantages accordés en \% de la colonne (6) par rapport à la colonne (5)

Pourcentage des importations en provenance des $\vec{\sigma} \vec{\sigma} \quad \vec{\sigma}$ PVD ayant effectivement bénéficié des préférences (6) en \% de (3) 
Les importations en provenance des pays en développement ont porté en 1982 sur 5.545 milliards de francs, soit $9,6 \%$ de l'ensemble des importations suisses, alors que les exportations à destination des

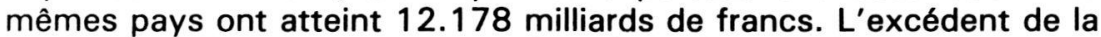
balance commerciale enregistré dans les échanges avec les pays en développement a donc été de 6.633 milliards de francs. Les importations ont légèrement baissé $(-2,3 \%)$, les exportations progressant de $3,8 \%$. La part prise par les pays en développement aux échanges avec la Suisse a continué de progresser, malgré le recul sensible enregistré par certains d'entre eux, comme le Brésil ou le Mexique, du fait du déséquilibre de leur balance des paiements.

Le 1er juillet 1982, le Conseil fédéral décida de faire bénéficier de l'exemption des droits de douane les 31 pays les moins avancés pour des produits industriels, qui, exportés à partir des autres pays en développement, jouiront d'une adaptation des préférences douanières; ces préférences seront également introduites pour quelques rares produits agricoles.

Au 1er janvier 1983, le Conseil fédéral a revu conformément à l'abaissement des droits de douane décidé par le GATT le taux des préférences douanières. A cette date entrait en vigueur la quatrième étape de la réduction des droits de douane décidée par le GATT à Tokio. Ces réductions annuelles sont échelonnées jusqu'en 1987. Pour la Suisse, le manque à gagner résultant de l'abaissement du taux des préférences douanières devrait se chiffrer à environ 0,5 million de francs pour 1983. On a continué à harmoniser au plan international la réglementation concernant l'origine des marchandises. Fin mai 1983, le Conseil fédéral a porté à 36 le nombre des pays les moins avancés appelés à profiter d'exemptions particulières dans le cadre général du système des préférences douanières. Ces 5 nouveaux pays figurant sur la liste sont la Guinée équatoriale, Djibouti, Sao-Tome et Principe, la SierraLeone et le Togo.

\section{Sources}

Bbl 1 1983, pp. 700 ss; Statistique du commerce extérieur de la direction générale des douanes de la Confédération, rapport annuel.

NZZ, 26.5.1983.

\section{ENTRETIENS ECONOMIQUES}

Entre juillet 1982 et juin 1983, plusieurs pays en développement et la Suisse ont eu des entretiens à caractère économique et commercial dont nous allons donner un aperçu.

A fin septembre, la Commission économique Suisse-République populaire de Chine a tenu à Berne sa 6ème réunion. Elle a examiné l'état 
actuel des échanges économiques et commerciaux entre les deux pays et leurs possibilités de développement. Elle a abordé entre autres des problèmes d'expansion commerciale et de coopération industrielle, y compris les «joint ventures». La délégation s'est également entretenue avec des représentants de l'industrie suisse. En 1982, les exportations directes de la Suisse vers la Chine ont atteint 263,3 millions de francs et les importations de la Suisse 146,3 millions de francs.

Une délégation économique suisse a participé à la 3ème réunion de la Commission économique mixte Inde-Suisse, du 16 au 18 février 1982 , à la Nouvelle-Delhi. Elle a décidé de désigner un groupe de travail appelé à se réunir deux fois par an au niveau des ambassadeurs. A ces réunions seront passés en revue l'état, les difficultés et les possibilités de développement des relations économiques et commerciales entre les deux pays.

L'Inde n'est pas pour la Suisse un partenaire commercial important, mais les exportations suisses (345,9 millions de francs en 1982) se sont accrues au cours des dernières années, surtout en biens d'inves tissement. Les importations en provenance de I'Inde ont aussi marqué une forte progression jusqu'en 1981 et se sont diversifiées (152 millions de francs en 1982).

Investissements directs et transferts de technologie sont les pièces maîtresses des relations économiques entre les deux pays. La Suisse figure au 4ème rang des pays qui investissent en Inde. De 1957 au premier trimestre de 1982, le gouvernement indien a approuvé plus de 380 contrats de coopération entre des entreprises industrielles indiennes et suisses. Après la Grande-Bretagne, les EU, la RFA et le Japon, la Suisse est au cinquième rang des fournisseurs de technologie de l'industrie indienne.

La Commission économique Suisse-Roumanie s'est réunie à Bucarest du 8 au 10 juin 1983 pour parler du commerce et de la coopération économique entre les deux pays. Elle a également passé en revue la situation économique des deux partenaires, la consolidation des dettes roumaines et le règlement des arriérés. Les exportations suisses en Roumanie ont atteint 78,4 millions de francs en 1982, les importations de Roumanie se chiffrant à 38,2 millions de francs.

Une délégation économique suisse conduite par le secrétaire d'Etat Jolles a séjourné du 26 au 31 mars 1983 en Egypte. Les entretiens ont porté sur des projets industriels et d'ingénierie suisse en Egypte. Vu les progrès de la coopération économique et l'augmentation des "joint ventures", on travaille à un accord de double imposition. Durant cette visite, les négociations concernant un nouveau crédit mixte d'un montant de 90 millions de francs ont abouti. Les échanges commerciaux entre l'Egypte et la Suisse sont à la hausse, notre pays enregistrant un excédent élevé (573,2 millions de francs aux exportations en 1982 contre 81,8 millions de francs aux importations). 
Du 18 au 28 février, une délégation économique suisse a séjourné en Jordanie et en Syrie pour procéder à un échange d'informations et œuvrer au développement des relations économiques entre ces deux pays et le nôtre.

Dans la deuxième quinzaine de mars, il y a eu des entretiens avec cinq pays d'Amérique latine sur les investissements et les échanges commerciaux avec la Suisse. Au Mexique, on a parlé de la situation économique actuelle qui a contraint à l'abandon de certains points du programme d'industrialisation, le commerce avec la Suisse étant également touché. On a aussi évoqué une reprise du paiement de ses dettes par le Mexique.

Au Panama, on a négocié un accord sur la protection des investissements entre le Panama et la Suisse, tout comme à la Barbade et à Trinité-et-Tobago. En parallèle à ces négociations bilatérales, l'ambassadeur Lévy a représenté la Suisse à la 24ème assemblée annuelle du Conseil des gouverneurs de la Banque interaméricaine de développement à Panama; il a rencontré à la Barbade des représentants de la Banque de développement des Caraïbes et il a examiné la possibilité de coopérer avec cette institution.

Le 10 janvier 1983, I'ambassadeur a participé à l'inauguration de I'exposition industrielle Swissexpo Far East à Hong-Kong. A cette occasion, il a parlé du développement des relations économiques mutuelles, tant dans le domaine commercial que pour les investissements directs et le transfert de technologie de pointe.

\section{Sources}

EVD, communiqués de presse, 28.9.1982; 14.2.1983.

$N Z Z, 21.2 .1983$.

EVD, communiqués de presse, $21,25.3,9.4,14.6 .1983$.

\section{EXPORTATIONS D'ARMES}

En 1982, les exportations suisses de matériel de guerre se sont élevées à 471,3 millions de francs (contre 511,5 millions de francs l'année précédente). Si elles ont un peu baissé en comparaison de 1981 , elles n'en ont pas moins conservé un volume important. Le matériel de guerre représente $0,89 \%$ de l'ensemble des exportations suisses en 1982 et la part de la Suisse aux exportations d'armes enregistrées dans le monde entier est de 0,4\%. Par groupes de pays, ce sont les pays en développement qui viennent en tête des acheteurs avec une part de $46 \%$ (42\% l'année précédente). La part des pays industriels a passé de $58 \%$ en 1981 à $54 \%$ en 1982 .

Cette progression des achats faits par des pays en développement s'explique par des ventes beaucoup plus volumineuses aux pays en 
développement du Sud-Est européen, c'est-à-dire la Grèce et la Turquie. Les exportations vers les pays de I'OPEP et les pays en développement situés hors de l'Europe ont nettement baissé. Le Nigeria reste quand même l'un des principaux clients. Des pays en développement comme I'Algérie, le Liberia, Singapour et la Turquie ont passé chacun des commandes allant de 1 à 10 millions de francs.

Aux termes de la loi, l'exportation de matériel de guerre est soumise à l'autorisation du Conseil fédéral. La loi sur les ventes de matériel de guerre ne concerne pas les fabrications sous licence à l'étranger et les exportations faites par des firmes suisses établies à l'étranger. L'exportation d'avions Pilatus, qui peuvent être dotés à l'étranger d'un équipement militaire, n'est pas soumise à la loi sur le matériel de guerre.

Au début de février 1983, les comités suisses de solidarité avec le Guatémala et I'association des Magasins du Monde ont transmis au Conseil fédéral une pétition portant 25.000 signatures. Ce document demande que les avions Pilatus-PC-7 et tout autre matériel de guerre soient frappés d'une interdiction d'exportation au Guatémala, car le gouvernement militaire de ce pays les utiliserait contre les populations. Le PCS s'est aussi inspiré de l'exemple guatémaltèque pour demander qu'on redéfinisse le terme de "matériel de guerre». Les exportations d'armes au Guatémala ont encore été critiquées par les conseillers nationaux Bäumlin (PS/BE) et Ziegler (PS/GE).

\section{Sources}

Institut d'éthique sociale, Berne.

TA, 3, 10.2, 24.6.1983. 


\section{EXPORTATIONS SUISSES DE MATERIEL DE GUERRE en 1982}

( en milliers de francs et en \%)

1. Total des exportations

471.305

100

2. vers les pays industriels

(non compris les pays à commerce étatisé)

253.626

54

3. vers les pays en développement ${ }^{*}$

au total

217.671

46

Pays en développernent sans l'OPEP

123.401

27

Pays en développement sans l'OPEP

ni les pays en dév. européens

9.205

2

répartition par groupes de pays

- Europe

118.196

Espagne

9.871

Grèce

98.613

Portugal

474

Turquie

9.131

autres (Yougoslavie, Chypre)

47

25

2

OPEP

Nigeria

90.270

87.882

21

Equateur

Algérie

autres (Arabie Séoudite, Qatar,

Emirats arabes, Koweit, Irak,

Iran, Indonésie, Vénézuela)

388

0

2

0

- Pays en développement à revenu élevé (HNV)

4.780

19

Singapour

Argentine

4.717

47

19

16

0

1.984

0

autres (Israel, Brunei, Antilles

néerlandaises)

16

0 
- Pays en développement à revenu moyen (MNV)

Pérou

Tunisie

864

0

Colombie

10

Oman

300

0

autres (Chili, Uruguay Nicaragua,

0

Brésil, Zimbabwe, Syrie, Liban,

Bahrein, Taiwan, Papousie-Nouvel-

le Guinée)

11

0

- Pays en développement à revenu bas (BNV)

$3.561 \quad 1$

- PMA

(Soudan, Botswana, Tanzanie, Rwanda)

- PPA

Inde

autres (Egypte, Pakistan, Côte d'Ivoire, Ghana, Kenya)

$\begin{array}{rl}11 & 0 \\ 3.424 & 1 \\ 612 & 0 \\ 0 & 0 \\ 2.809 & 1 \\ 3 & 0\end{array}$

- Autres BNV

Maroc

Philippines

Liberia autres (Jordanie, Thailande)

* Répartition des pays en développement et adoption des critères pour la formation des groupes, selon Höhn, Der Schweizerische Aussenhandel mit der Dritten Welt, Adliswil 1980, p. 19 ss. et annexe.

Figurent dans la liste tous les pays ayant acheté en l'espace d'un an pour Fr. 100.000 au moins, valeur à l'exportation. Les autres sont nommés, mais classés sous la dénomination "autres". 